Rev. Biol. Neotrop. 5(2): 1-9, 2008

\title{
STRUTURA E DiSTRIBUição ESPACIAL DE TRICHILIA ELEGANS A. JuSS. (Meliaceae) em uma floresta semidecídua no Pantanal da Nhecolândia, Mato Grosso do Sul, Brasil
}

\section{Carlos Rodrigo Lehn}

Portal Educação, Rua 7 de Setembro 1686, Centro, 79002-130, Campo Grande, Mato Grosso do Sul, Brasil; e-mail: crlehn@gmail.com

\author{
Suzana Maria Salis \\ Embrapa Pantanal, Caixa Postal 109, 79320-900, Corumbá, Mato Grosso do Sul, Brasil
}

\section{Patrícia Póvoa Mattos}

Embrapa Florestas, Caixa Postal 319, 83411-000, Colombo, Paraná, Brasil

\section{Geraldo Alves Damasceno Junior}

Departamento de Biologia - CCBS, Universidade Federal de Mato Grosso do Sul, Av. Senador Filinto Muller, s/no, Cidade Universitária, 79070-900, Campo Grande, Mato Grosso do Sul, Brasil

\begin{abstract}
Resumo: Trichilia elegans A. Juss. é uma espécie com ampla distribuição, que ocorre desde a Venezuela até o Uruguai, e constitui componente comum no sub-bosque de formações estacionais. Mesmo que os primeiros estudos sobre a flora pantaneira datem de mais de um século, trabalhos voltados para a estrutura e a distribuição espacial de espécies arbóreas ocorrentes na região são ainda raros. Os objetivos do presente estudo foram identificar o padrão de distribuição espacial e a distribuição diamétrica de T. elegans e verificar se existe correlação entre o número de indivíduos jovens e o número de indivíduos adultos em uma floresta semidecídua no Pantanal da Nhecolândia. Para o levantamento, foram amostradas 50 parcelas de $200 \mathrm{~m}^{2}$ cada e inventariados os indivíduos jovens e adultos em cada uma delas. Com o intuito de identificar o padrão de distribuição espacial, foram utilizados o Índice de Morisita (IM) e a razão de variância/média (R). Foram encontrados 218 indivíduos jovens e 78 adultos. Indivíduos jovens e adultos apresentaram distribuição espacial agrupada, estando os jovens (IM $=2,32 ; R=5,49$ ) mais agrupados que os adultos ( $\mathrm{IM}=1,88 ; \mathrm{R}=2,05$ ). O coeficiente de correlação linear encontrado $(r=0,60)$ demonstrou que a densidade de indivíduos jovens está altamente correlacionada com a densidade de indivíduos adultos.
\end{abstract}

Palavras-chave: Estádios ontogenéticos, estrutura, Meliaceae, padrão espacial, Pantanal.

ABstract: Trichilia elegans A. Juss. is a widely distributed species that occurs from Venezuela to Uruguay and constitutes a common component of seasonal forests. Even though the studies on the Pantanal flora started last century, researches on the structure and spatial distribution of tree species are still rare. This work aimed at identifying the pattern of spatial distribution and the diametric distribution of $T$. elegans and verifying whether there is a correlation between the number of young individuals and the number of adult individuals in a semi-deciduous forest of the Pantanal da Nhecolândia. We sampled 50 plots of $200 \mathrm{~m}^{2}$ each and registered the young and adult individuals in each one of them. To identify the pattern of spatial distribution, we used the Morisita Index (MI) and the variance/mean ratio (R). The linear correlation coefficient was used to verify whether there is a correlation between populations of the juveniles and adults. We found 218 young and 78 adult individuals. Young and adult individuals presented gregarious spatial distribution, and the young ones $(\mathrm{MI}=2.32 ; \mathrm{R}=5.49)$ were more gregarious than the adults ( $M I=1.88 ; R=2.05)$. The linear correlation coefficient found $(r=0.60)$ demonstrated that the density of young individuals is highly correlated with the density of adult individuals.

KeY words: Ontogenetic stages, structure, Meliaceae, spatial pattern, Pantanal. 


\section{INTRODUÇÃO}

U ma população compreende os indivíduos de uma espécie dentro de uma dada área, apresentando comportamento dinâmico e mudando com o tempo em decorrência de recrutamento e mortalidade.

O arranjo dos membros de uma população é conhecido como padrão de distribuição espacial, podendo os indivíduos apresentar padrão de distribuição espacial: aleatório quando distribuídos ao acaso; regular - quando em intervalos similares; e agregada - no caso da formação de manchas (Meirelles \& Luiz, 1995). Este comportamento é resultante da ação conjunta de fatores bióticos e abióticos, como competição por espaço, disponibilidade de nutrientes, luz e água sobre os processos de recrutamento e mortalidade. Trata-se de uma informação muito importante, podendo ser utilizada em estudos sobre regeneração natural, dinâmica pós-distúrbios (Vacek \& Lepš, 1996) e relações ecológicas entre espécies, tais como competição (Duncun, 1991) e dispersão (Collins \& Klahr, 1991).

Atualmente, uma das maiores preocupações para a conservação das florestas tropicais é a transformação e a exploração deste habitat. A exploração modifica severamente a estrutura do habitat (Lieberman \& Dock, 1982), atuando diretamente no padrão de distribuição espacial (Almeida et al., 1998) e fenológico das espécies nativas (Costa \& Magnusson, 2003), já que uma série de fatores (luz incidente, umidade, disponibilidade de nutrientes) também será afetada.

O manejo das florestas tropicais, em sua essência, para a utilização de seus recursos de forma sustentável e, como consequência, para a sua conservação, depende da compreensão da dinâmica das populações (Van Groenendael et al., 1996) mediante a realização de estudos que caracterizem principalmente a estrutura populacional das espécies (Nascimento et al., 2001).

Estudos voltados para a distribuição espacial e/ou aspectos estruturais de populações de comunidades arbóreas foram realizados em diversas regiões do Brasil, como Centro-Oeste (Dubs, 1992), Sudeste (Antonini \&
Nunes-Freitas, 2004; Carvalho et al., 2000; Espírito-Santo et al., 2002; Ferreira-Júnior et al., 2007; Souza et al., 2007); Sul (Bianchini et al., 2003; Giehl et al., 2007; Lindenmaier \& Budke, 2006), bem como em países da América do Sul, como a Venezuela (Kammescheidt, 1998) e a Bolívia (Killeen et al., 1998), e da Ásia, como a Malásia (Seidler \& Plotkin, 2006).

No Pantanal, as formações florestais vêm sendo exploradas há mais de dois séculos, principalmente pela retirada seletiva de madeira para a construção de casas, cercas e currais. Apesar dos primeiros estudos sobre a flora pantaneira datarem de mais de um século, estudos voltados para a estrutura e a distribuição espacial das espécies arbóreas ocorrentes na região ainda são escassos.

Meliaceae é uma família com distribuição pantropical, incluindo cerca de 50 gêneros e 600 espécies (Pennington, 1981). No Brasil, ocorrem seis gêneros e cerca de 100 espécies. Entre os gêneros com ocorrência no Brasil, Trichilia caracteriza-se por apresentar espécies de médio a grande porte, sendo um componente muito comum no sub-bosque de florestas estacionais (Souza \& Lorenzi, 2005).

Trichilia elegans A. Juss. é uma espécie de ampla dispersão, encontrada desde a Venezuela até o Uruguai, e que no Pantanal ocorre preferencialmente em áreas de mata semidecídua com solos férteis. Trata-se de uma espécie muito abundante no sub-bosque de áreas florestadas situadas no Pantanal da Nhecolândia e que, historicamente, tem sido utilizada para marcenaria e carpintaria por apresentar madeira resistente e durável, sendo sua casca utilizada como corante em processos de tingimento (Pott \& Pott, 1994). Entretanto, pouco se conhece acerca dos aspectos estruturais de populações desta espécie situadas no Pantanal.

Dessa forma, o objetivo deste trabalho foi analisar a estrutura e identificar o padrão de distribuição espacial de T. elegans e verificar se existe correlação entre a densidade de indivíduos jovens e a densidade de indivíduos adultos dessa espécie em uma floresta semidecídua situada no Pantanal da Nhecolândia, estado de Mato Grosso do Sul, Brasil. 


\section{Material e Métodos}

\section{Caracterização da ÁREA de estudo}

$\mathrm{O}$ presente estudo foi realizado na $\mathrm{Fa}-$ zenda Nhumirim (18 $59^{\prime} \mathrm{S}$ e 56 $\left.58^{\circ} \mathrm{W}\right)$, de propriedade da Embrapa Pantanal, sub-região da Nhecolândia, município de Corumbá, Mato Grosso do Sul (Figura 1). A vegetação da fazenda é composta por 52\% de campo, 20,7\% de cerradão, 6,6\% de floresta semidecídua e 20,5\% de lagoas e salinas (Embrapa, 1994).

Os dados foram coletados em fevereiro de 2006 em uma cordilheira com floresta semidecídua. As cordilheiras são áreas elevadas do relevo, situadas entre $2 \mathrm{~m}$ a $5 \mathrm{~m}$ acima dos campos alagáveis. Segundo Silva et al. (2000), as cordilheiras são diques marginais originados a partir da deposição aluvial de antigos rios, com extensão variando de um a vários quilômetros de comprimento, e cerca de $80 \mathrm{~m}$ a $300 \mathrm{~m}$ de largura. Sobre as cordilheiras encontra-se vegetação de cerrado, cerradão ou mata (Ratter et al., 1988). A maioria das fisionomias florestadas do Pantanal situa-se em áreas de cordilheira (Fernandes et al., 1999), que dificilmente sofrem alagamento durante o período das cheias.

O clima da região, segundo o sistema de classificação de Köppen, é do tipo $A w$, tropical megatérmico, com a temperatura média do mês mais frio superior a $18^{\circ} \mathrm{C}$, com inverno seco e chuvas no verão. As médias anuais de precipitação e temperatura no período 1977-1995 foram de $1.182,7 \mathrm{~mm}$ e $25,5^{\circ} \mathrm{C}$, respectivamente (Soriano, 1999). A maior precipitação média geralmente ocorre em janeiro, com 216,8 mm e a menor, em julho, com 19,7 mm (Soriano, 2002). As temperaturas máximas absolutas podem ultrapassar $40^{\circ} \mathrm{C}$ nos meses de outubro a janeiro (Embrapa, 1997). A região pode apresentar deficiência hídrica anual superior a $300 \mathrm{~mm}$, principalmente nos meses de agosto a outubro (Soriano, 1999).

De acordo com Ratter et al. (1988), os solos das cordilheiras da Fazenda Nhumirim são ricos em cálcio, com níveis de contração similares aos observados nas matas secas decíduas e florestas semidecíduas do Brasil Central.

\section{Coleta dos dAdOS}

A área amostrada perfaz um total de um hectare (200 m x $50 \mathrm{~m}$ ), a qual foi subdividida em 50 parcelas de $200 \mathrm{~m}^{2}$ cada. As parcelas foram montadas a aproximadamente $30 \mathrm{~m}$ da borda da cordilheira, com dimensões de $10 \mathrm{~m} \times 20 \mathrm{~m}$, tendo sido amostrados todos os indivíduos de T. elegans que apresentavam diâmetro à altura do peito $(\mathrm{DAP}) \geq 1 \mathrm{~cm}$. Foram considerados jovens todos os indivíduos que apresentavam DAP $\leq 3 \mathrm{~cm}$, delimitação adotada com base em observações de campo e de acordo com Gondim (2001).

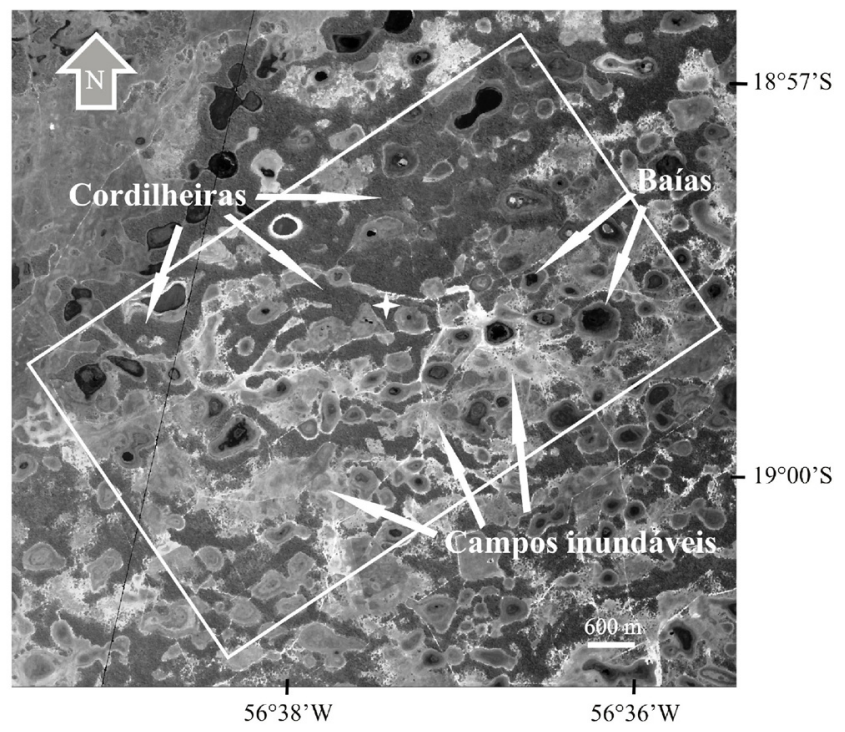

Figura 1 - Vegetação da Fazenda Nhumirim, Corumbá, Mato Grosso do Sul, indicando o local da área de estudo (estrela). 


\section{ANÁLISE ESTATÍSTICA}

O padrão de distribuição espacial foi analisado com base no Índice de Morisita (IM), sendo também estabelecida a razão variância/média (R) (Zar, 1999). Nos dois métodos, valores menores que 1,0 indicam ausência de agrupamento, valores iguais a 1,0 indicam distribuição uniforme e valores maiores que 1,0 indicam distribuição agregada. A significância estatística foi verificada através do valor de $\chi^{2}$ (qui-quadrado), com significância a 5\%. Para verificar a existência de correlação entre as populações de jovens e adultos, utilizou-se o coeficiente de correlação linear (r) (Zar, 1999) e a significância do valor obtida pelo teste $\mathrm{t}$ (Brower \& Zar, 1984).

A razão $\mathrm{R}$ foi calculada pela fórmula:

$$
\mathrm{R}=\mathrm{S}^{2} / \xi
$$

sendo:

$\mathrm{S}^{2}=$ variância

$\xi=$ média de indivíduos por parcela.

O Índice de Morisita (IM) foi obtido por:

$$
\mathrm{IM}=\mathrm{n} \cdot\left(\sum \mathrm{x}^{2}-\mathrm{N} / \mathrm{N}(\mathrm{N}-1)\right)
$$

sendo:

$\mathrm{n}$ = número total de parcelas amostradas;

$\sum \mathrm{x}^{2}=$ soma do quadrado do número de indivíduos por parcela;

$\mathrm{N}$ = número total de indivíduos amostrados em todas as parcelas.

A significância dos valores de $\mathrm{R}$ e IM foi verificada pelo teste do qui-quadrado $\left(\chi^{2}\right)$, calculando-se:

$$
\chi^{2}=\mathrm{n} \cdot\left(\sum \mathrm{x}^{2} / \mathrm{N}\right)-\mathrm{N}
$$

\section{ANÁLISE ESTRUTURAL}

Os diâmetros dos indivíduos observados foram distribuídos em classes com intervalos de $3 \mathrm{~cm}$. Foi calculado o quociente q de Liocourt, obtido dividindo-se cada classe diamétrica pela anterior (Meyer, 1952), permitindo fazer inferências sobre o recrutamento e a mortalidade em florestas naturais. Uma razão constante entre as classes indica que a taxa de recrutamento é similar à taxa de mortalidade, entendendo-se, assim, que a distribuição pode ser considerada regular ou equilibrada (Nascimento et al., 2004).

\section{Resultados e Discussão}

\section{DisTRIBUIÇÃO ESPACIAL}

Foram encontrados 218 indivíduos jovens e 78 adultos, obtendo-se uma população amostrada de 296 indivíduos (Figura 2). O número de indivíduos adultos variou de zero a 10 por parcela (Figura 2). Esta diferença resultou em IM $=1,88$ e $\mathrm{R}=2,05$, indicando distribuição espacial agregada. $\mathrm{O}$ número de indivíduos jovens variou muito entre parcelas, situando-se entre zero e 26 indivíduos, resultando em IM = 2,32 e R=5,49, o que também indica padrão de distribuição espacial agregado para os indivíduos jovens. A significância estatística observada pelo valor de $\chi^{2}=116,87$ para adultos e $\chi^{2}=445,87$ para jovens foi, para ambos os índices, maior que o esperado.

De acordo com Antonini \& Nunes-Freitas (2004), o padrão agregado de distribuição espacial é característico de espécies vegetais dispersas por animais, como é o caso de T. elegans. $\mathrm{O}$ conceito de sombras de sementes (seed shadows) demonstra que muitas espécies ornitocóricas

Jovens

\begin{tabular}{|c|c|c|c|c|c|c|c|c|c|}
\hline 6 & 1 & 1 & 3 & 1 & 1 & 5 & 3 & 9 & 1 \\
\hline 5 & 4 & 4 & 6 & 4 & 2 & 1 & & 9 & 26 \\
\hline 18 & 5 & 5 & 8 & & 14 & 12 & 1 & 10 & 2 \\
\hline 1 & 1 & 1 & 3 & & 6 & 9 & 1 & 1 & 2 \\
\hline 2 & 1 & 4 & 4 & 6 & 1 & 5 & & & \\
\hline
\end{tabular}

Adultos

\begin{tabular}{|l|l|l|l|l|l|l|l|l|l|}
\hline 3 & 1 & & & 1 & 1 & & 2 & 1 & \\
\hline & 1 & & 1 & 1 & & & 1 & 1 & 10 \\
\hline 4 & 3 & 3 & 4 & 2 & 4 & 3 & & 1 & \\
\hline & 2 & 2 & 2 & 1 & 1 & 1 & 7 & 4 & 1 \\
\hline & & & 2 & 1 & & 1 & 1 & 1 & 1 \\
\hline
\end{tabular}

Figura 2 - Número de indivíduos jovens e adultos de Trichilia elegans A. Juss. amostrados em cada uma das 50 parcelas (200 $\mathrm{m}^{2}$ cada) em floresta semidecídua, na Fazenda Nhumirim, Pantanal da Nhecolândia, Mato Grosso do Sul. 
são agrupadas pelo fato de suas sementes serem disseminadas predominantemente sob ninhos ou ao longo de rotas utilizadas pelas aves (Schupp et al., 2002). Outro aspecto relevante a ser observado é que as sementes de T. elegans germinam em locais sombreados, fator este que, aliado à descontinuidade do dossel, pode, de certa forma, influenciar no padrão de distribuição da espécie.

Estudos realizados em áreas florestais indicam padrão agregado de distribuição espacial para espécies arbóreas, entre os quais destacam-se os trabalhos de Kammescheidt (1998), que estudou uma formação florestal decídua na Reserva de Caparo, na Venezuela, Condit et al. (2000), que estudaram seis áreas florestais incluindo desde florestas decíduas secas a formações perenes situadas no Panamá e na Malásia, e ainda Lindenmaier \& Budke (2006) e Giehl et al. (2007), que analisaram a distribuição espacial de espécies arbóreas situadas em duas florestas estacionais no Rio Grande do Sul.

Analisando-se as populações de jovens e adultos, constatou-se que os indivíduos jovens se encontram mais agrupados na área estudada, indicando que o grau de agregação tendeu a diminuir durante o desenvolvimento ontogenético.

A diminuição da agregação ao longo do desenvolvimento ontogenético tem sido comumente interpretada como um indicativo da ocorrência de mecanismos dependentes de densidade e/ou distância (Hutchings, 1986). Conforme Solbrig (1981), a maioria das populações de espécies tropicais tem mortalidade concentrada nas classes de menor diâmetro. Dessa forma, populações em estádios ontogenéticos posteriores poderiam estar menos agrupadas, caso nem todos estes indivíduos jovens conseguissem atingir sua maturidade, por alguma razão qualquer.

Marques \& Joly (2000) observaram agregação em todas as classes de tamanho em uma população de Calophyllum brasiliense Camb. (Clusiaceae). De acordo com Clark \& Clark (1984), a distribuição espacial dos indivíduos e as distâncias de recrutamento não são constantes com o tempo e é o acúmulo destas modificações que determina o padrão de distribuição espacial dos adultos. Da mesma forma que no presente estudo, Oliveira et al. (1989) relataram maior agregação nos indivíduos jovens para uma população de Kielmeyera coriacea Mart. (Clusiaceae) no Distrito Federal.

\section{Correlação entre jovens e adultos}

O coeficiente de correlação linear encontrado entre a densidade de indivíduos jovens e a densidade de indivíduos adultos foi de 0,60 (Figura 3). Este resultado foi considerado significativo ( $\mathrm{p}<0,001)$, indicando que a densidade de indivíduos jovens está altamente associada ao número de indivíduos adultos.

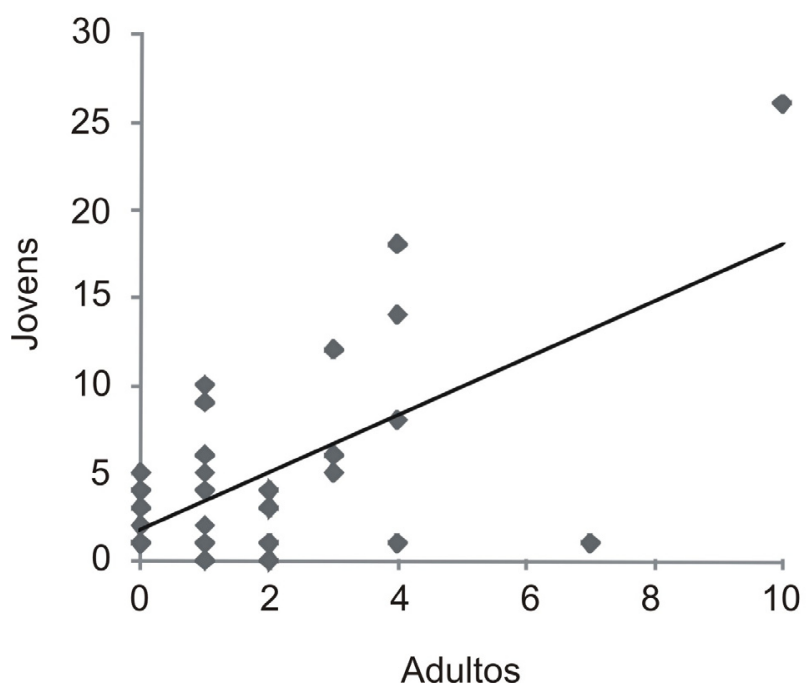

Figura 3 - Correlação entre a densidade de indivíduos jovens e adultos de Trichilia elegans A. Juss. em floresta semidecídua, na Fazenda Nhumirim, Pantanal da Nhecolândia, Mato Grosso do Sul, Brasil. Cada ponto no gráfico representa o número de indivíduos adultos e jovens em uma determinada parcela. 
Correlações positivas entre dois estádios ontogenéticos podem indicar que a variação positiva da densidade de indivíduos adultos ocasiona aumento na densidade de indivíduos jovens, o que pode influenciar diretamente no padrão de distribuição espacial apresentado pelas espécies (Budke et al., 2004; Hutchings, 1986; Leirana-Alcocer \& Parra-Tabla, 1999).

Ao estudar uma população de Calophyllum brasiliense, Marques \& Joly (2000) observaram que as densidades de indivíduos jovens e subadultos estavam fortemente correlacionadas. Entretanto, no mesmo estudo, quando os autores levaram em consideração os indivíduos jovens e adultos, não observaram correlação, relatando ainda maior mortalidade nas parcelas em que detectaram maior número de indivíduos subadultos. Conforme já salientado, a maioria das populações de espécies tropicais tem mortalidade concentrada nas classes mais jovens (Solbrig, 1981), o que poderia explicar o menor número de indivíduos adultos na população de T. elegans estudada.

\section{ESTRUTURA}

A análise da estrutura diamétrica revelou maior número de indivíduos nas classes de menor diâmetro (Figura 4), apresentando esta população distribuição de diâmetros ten- dendo ao padrão de "J" invertido. Os valores calculados para o quociente de Liocourt variaram de $\mathrm{q} 1=0,23$ a q5 $=0,66$, estando a maior parte dos valores situados entre 0,23 e 0,50.

Distribuições diamétricas equilibradas, nas quais o recrutamento compensa a mortalidade ao longo do tempo, indicando um padrão de distribuição dos diâmetros tendendo ao padrão de "J" invertido, apresentam valores relativamente constantes de q (Meyer et al., 1961; Nascimento et al., 2004), o que não parece ser o caso da população estudada. O elevado número de indivíduos observado nas classes de menor diâmetro indica que a espécie possui grande potencial regenerativo e de manutenção dos níveis atuais de densidade. Entretanto, poucos indivíduos estão conseguindo atingir as classes diamétricas posteriores.

Killeen et al. (1998) observaram padrão similar de distribuição diamétrica para uma população de T. elegans situada em uma floresta semidecídua na região da Chiquitania, na Bolívia. Para Felfili (1997), algumas espécies requerem escalas espacial e temporal muito amplas para atingir certo equilíbrio entre mortalidade e recrutamento, principalmente em populações que tenham sofrido algum distúrbio. Felfili (1995) comentou que estas variações são geralmente relacionadas à

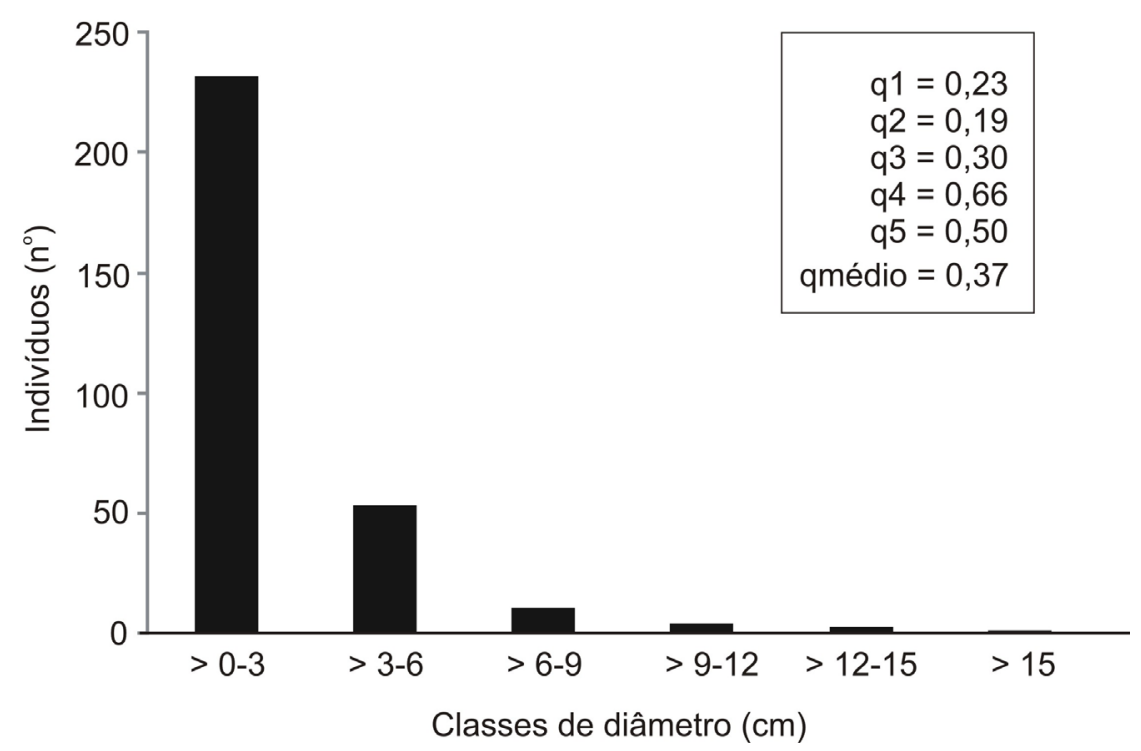

Figura 4 - Distribuição diamétrica de uma população de Trichilia elegans A. Juss. situada em floresta semidecídua, na Fazenda Nhumirim, Pantanal da Nhecolândia, Mato Grosso do Sul, Brasil. 
ecologia populacional de cada espécie e que, na maioria dos casos, observa-se que existem grandes descontinuidades ou achatamentos nas distribuições diamétricas.

A análise dos dados aqui apresentados permite-nos concluir que a população estudada apresenta elevado potencial de autorregeneração, uma vez que existe a possibilidade de substituição natural dos indivíduos mortos de classes diamétricas maiores pelo recrutamento dos indivíduos abundantes das classes diamétricas inferiores.

Estudos voltados para a distribuição espacial e a estrutura de espécies arbóreas no Pantanal são ainda escassos, o que, por sua vez, torna difícil a comparação e mesmo o pleno entendimento sobre a biologia dessas espécies. No que diz respeito a T. elegans, um estudo com período de execução a longo prazo seria extremamente oportuno para que esta dinâmica possa ser melhor compreendida.

\section{REFERÊNCIAS}

Almeida, D. R., L. Cogliatti-Carvalho \& C. F. D. Rocha. 1998. As bromeliáceas da Mata Atlântica da Ilha Grande, RJ: composição e diversidade de espécies em três ambientes diferentes. Bromélia 5: 54-65.

Antonini, R. D. \& A. F. Nunes-Freitas. 2004. Estrutura populacional e distribuição espacial de Miconia prasina DC. (Melastomataceae) em duas áreas de Floresta Atlântica na Ilha Grande, RJ, Sudeste do Brasil. Acta Bot. Bras. 18: 671-676.

Bianchini, E., R. S. Popolo, M. C. Dias \& J. A. Pimenta. 2003. Diversidade e estrutura de espécies arbóreas em área alagável do município de Londrina, Sul do Brasil. Acta Bot. Bras. 17: 405-419.

Brower, J. E. \& J. H. Zar. 1984. Field \& laboratory methods for general ecology. 2nd ed., Wm. C. Publishers, Dubuque, $226 \mathrm{p}$.

Budke, J. C., E. L. H. Giehl, E. A. Athayde \& R. A. Záchia. 2004. Distribuição espacial de Mesadenella cuspidata (Lindl.) Garay (Orchidaceae) em uma floresta ribeirinha em Santa Maria, RS, Brasil. Acta Bot. Bras. 18: 31-35.
Carvalho, M. T. L., M. A. L. Fontes \& A. T. Oliveira-Filho. 2000. Tree species distribution in canopy gaps and mature forest in an area of cloud forest of the Ibitipoca Range, south-eastern Brazil. Pl. Ecol. 149: 9-22.

Clark, D.A. \& D. B. Clark. 1984. Spacing dynamics of a tropical rain forest tree: evaluation of the Janzen Connell model. Am. Nat. 124: 769-788.

Collins S. L. \& S. C. Klahr. 1991. Tree dispersion in oak-dominated forest along an environmental gradient. Oecologia 86: 471-477.

Condit, R., P. S. Ashton, P. Baker, S. Bunyavejchewin, S. Hubbell, R. B. Foster, A. Itoh, J. V. LaFrankie, H. S. Lee, E. Losos, N. Manokaran, R. Sukumar \& T. Yamakura. 2000. Spatial patterns in the distribution of tropical tree species. Science 288: 1414-1418.

Costa, F. R. C. \& W. E. Magnusson. 2003. Effects of selective logging on the diversity and abundance of flowering and fruiting understory plants on a Central Amazonian Forest. Biotropica 35: 103-114.

Dubs, B. 1992. Observations on the differentiation of woodland and wet savanna habitats in the Pantanal of Mato Grosso, Brazil, p. 431-449. In: P. A. Furley, J. Proctor \& J. A. Ratter (Eds), Nature and dynamics of forest savanna boundaries. London, Chapman \& Hall.

Duncun R. D. 1991. Competition and the coexistence of species in a mixed podocarp stand. J. Ecol. 79: 1073-1084.

Embrapa. 1994. Plano de manejo da Estação Ecológica Nhumirim. Centro de Pesquisa Agropecuária do Pantanal (Corumbá, MS). EMBRAPA-CPAP, Corumbá, 94 p. (Documentos 12). Disponível em: <http:// www.cpap.embrapa.br/publicacoes/ online/DOC12.pdf>. Acesso em: 5 mar. 2009.

Embrapa. 1997. Boletim agrometeorológico: 1986 - 1996 (Fazenda Nhumirim). EMBRAPA CPAP, Corumbá. Boletim agrometeorológico 3. Disponível em: <http:/ / www.cpap.embrapa.br/publicacoes/online/BA03.pdf> Acesso em: 5 mar. 2009. 
Espírito-Santo, F. D. B., A. T. Oliveira-Filho, E. L. M. Machado, J. S. Souza, M. A. L. Fontes \& J. J. G. S. M. Marques. 2002. Variáveis ambientais e distribuição de espécies arbóreas em um remanescente de floresta estacional semidecídua montana no campus da Universidade de Lavras, MG. Acta Bot. Bras. 16: 331-356.

Felfili, J. M. 1995. Diversity, structure and dynamics of a community forest in central Brazil. Vegetatio 117: 1-15.

Felfili, J. M. 1997. Diameter and height distributions in a gallery forest community and some of its main species in central Brazil over a six-year period (1985-1991). Rev. Brasil. Bot. 20: 155-162.

Fernandes, F. A., C. C. Cerri \& A. H. B. M. Fernandes. 1999. Alterações na matéria orgânica de um podzol hidromórfico pelo uso com pastagens cultivadas no pantanal mato-grossense. Pesq. Agropec. Bras. 34: 1943-1951.

Ferreira-Júnior, W. G., A. F. Silva, C. E. G. R. Schaefer, J. A. A. M. Neto, A. S. Dias, M. Ignácio \& M. C. M. P. Medeiros. 2007. Influence of soils and topographic gradients on tree species distributions in a Brazilian Atlantic tropical forest. Edinb. J. Bot. 64: 137-157.

Giehl, E. L. H., J. C. Budke \& E. A. Athayde. 2007. Distribuição espacial de espécies arbóreas em uma floresta estacional em Santa Maria, sul do Brasil. Pesq. Bot. 58: 215-226.

Gondim, M. J. C. 2001. Dispersão de sementes de Trichilia spp. (Meliaceae) por aves em um fragmento de mata mesófila semidecídua, Rio Claro, SP, Brasil. Ararajuba 9: 101-112.

Hutchings, M. J. 1986. The structure of plant population, p. 97-136. In: M. J. Crawley (Ed), Plant ecology. Blackwell Scientific Publications, Oxford.

Kammescheidt, L. 1998. Stand structural and spatial pattern of commercial species in logged and unlogged Venezuelan Forest. For. Ecol. Manag. 109: 163-174.

Killeen, T. J., A. Jardim, F. Mamani \& N. Rojas. 1998. Diversity, composition and structure of a tropical semideciduous forest in the Chiquitania Region of Santa Cruz, Bolivia. J. Trop. Ecol. 14: 803-827.

Leirana-Alcocer, J. \& V. Parra-Tabla. 1999. Factors affecting the distribution, abundance, and seedling survival of Mammillaria gaumeri, an endemic cactus of coastal Yucatán, México. J. Arid Environ. 41: 421-428.

Lieberman, S. S. \& C. F. Dock. 1982. Analysis of the leaf litter arthropod fauna of a lowland tropical evergreen forest site (La Selva, Costa Rica). Rev. Biol. Trop. 30: 27-34.

Lindenmaier, D. S. \& J. C. Budke. 2006. Florística, diversidade e distribuição espacial das espécies arbóreas em uma floresta estacional na bacia do Rio Jacuí, Sul do Brasil. Pesq. Bot. 57: 193-216.

Marques, M. C. M. \& C. A. Joly. 2000. Estrutura e dinâmica de uma população de Calophyllum brasiliense Camb. em floresta higrófila do sudeste do Brasil. Rev. Brasil. Bot. 23: 107-112.

Meirelles, M. L. \& A. J. B. Luiz. 1995. Padrões espaciais de árvores de um cerrado em Brasília, DF. Rev. Brasil. Bot. 18: 185-189.

Meyer, H. A. 1952. Structure, growth, and drain in balanced unevenaged forests. J. For. 50: 85-92.

Meyer, H. A., A. B. Recknagel, D. D. Stevensoon \& R. A. Bartoo. 1961. Forest management. Belhaven Press, Cambridge, 282 p.

Nascimento, A. R. T., J. M. Felfili \& E. M. Meirelles. 2004. Florística e estrutura da comunidade arbórea de um remanescente de floresta estacional decidual de encosta, Monte Alegre, GO, Brasil. Acta Bot. Bras. 18: 659-669.

Nascimento, N. A., J. O. P. Carvalho \& N. V. M. Leão. 2001. Distribuição espacial de espécies arbóreas relacionada ao manejo de florestas naturais. Rev. Ciênc. Agr. 37: 175-194.

Oliveira, P. E. A. M., J. F. Ribeiro \& M. I. Gonzales. 1989. Estrutura e distribuição espacial de uma população de Kielmeyera coriacea Mart. de cerrados de Brasília. Rev. Bras. Bot. 12: 39-47.

Pennington, T. D. 1981. Meliaceae. In: Flora Neotropica. Monographie 28: 1-472. 
Pott, A. \& V. J. Pott. 1994. Plantas do Pantanal. Embrapa-SPI, Embrapa-CPAP, Brasília, DF, 320 p.

Ratter, J. A., A. Pott, V. J. Pott, C. N. Cunha \& M. Haridassan. 1988. Observations on woody vegetation types in the Pantanal around Corumbá. Notes Royal Bot. Garden Edinb. 45: 503-525.

Schupp, E. W., T. Milleron \& S. Russo. 2002. Dissemination limitation and the origin and maintenance of species-rich tropical forests, p. 19-33. In: D. J. Levey, W. R. Silva \& M. Galleti (Eds), Seed dispersal and frugivory: ecology, evolution and conservation. CABI Publishing, New York.

Seidler, T. G. \& J. B. Plotkin. 2006. Seed dispersal and spatial pattern in tropical trees. PLoS Biol. 4: 2132-2137.

Silva, M. P., V. J. Pott, F. J. Ponzoni \& A. Pott. 2000. Fitossociologia e estrutura de cerradão e mata semidecídua do Pantanal da Nhecolândia. In: Anais do III Simpósio sobre Recursos Naturais e SócioEconômicos do Pantanal: Os desafios do Novo Milênio. Embrapa Pantanal, Corumbá. Disponível em: <http://www.cpap. embrapa.br/agencia/congresso/Bioticos/ SILVA-082.pdf>. Acesso em: 5 mar. 2009.

Solbrig, O. T. 1981. Studies on the population biology of the genus Viola. II. The effect of plant size on fitness in Viola sororia. Evolution 35: 1080-1093.
Soriano, B. M. A. 1999. Caracterização climática de Corumbá. EMBRAPA-CPAP, Corumbá, 25 p. (Boletim de Pesquisa 11). Disponível em: <http://www.cpap.embrapa.br/publicacoes/online/BP11.pdf $>$. Acesso em: 5 mar. 2009.

Soriano, B. M. A. 2002. Boletim agrometeorológico: 1999 (Fazenda Nhumirim). EMBRAPA-CPAP, Corumbá, 21 p. (Documentos 26). Disponível em: <http:// www.cpap.embrapa.br/publicacoes / online/DOC26.pdf>. Acesso em: 5 mar. 2009.

Souza, J. P., G. M. Araújo \& M. Haridasan. 2007. Influence of soil fertility on the distribution of tree species in a deciduous forest in the Triângulo Mineiro region of Brazil. Plant Ecol. 191: 253-263.

Souza, V. C. \& H. Lorenzi. 2005. Botânica Sistemática. Editora Instituto Plantarum, Nova Odessa, 640 p.

Vacek, S. \& J. Lepš. 1996. Spatial dynamics of forest decline: the role of neighboring trees. J. Veget. Sci. 7: 789-798.

Van Groenendael, J. M., S. H. Bullock \& L. A. Pérez-Jiménez. 1996. Aspects of population biology of gregarious tree Cordia elaegnoides in Mexican tropical deciduous Forest. J. Trop. Ecol. 12: 11-24.

Zar, J. H. 1999. Bioestatistical analysis. 4th ed., Prentice Hall, Upper Saddle River, $663 \mathrm{p}$. 